\title{
A comparison of the molecular subtypes of triple-negative breast cancer among non-Asian and Taiwanese women
}

\author{
Ling-Ming Tseng ${ }^{1,2} \cdot$ Jen-Hwey Chiu ${ }^{1,3,4} \cdot$ Chun-Yu Liu $^{5} \cdot$ Yi-Fang Tsai ${ }^{1,6}$. \\ Yun-Lin Wang ${ }^{3} \cdot$ Chu-Wen Yang ${ }^{7} \cdot$ Yi-Ming Shyr ${ }^{1}$
}

Received: 15 February 2017/ Accepted: 8 March 2017/Published online: 15 March 2017

(C) The Author(s) 2017. This article is published with open access at Springerlink.com

\begin{abstract}
Background "Precision medicine" is a concept that by utilizing modern molecular diagnostics, an effective therapy is accurately applied for each cancer patient to improve their survival rates. The treatment of triple-negative breast cancer (TNBC) remains a challenging issue. The aim of this study was to compare the molecular subtypes of triplenegative breast cancer (TNBC) between Taiwanese and Non-Asian women.

Methods GEO Datasets for non-Asian (12 groups, $n=1450$ ) and Taiwanese (3 groups, $n=465$ ) breast cancer, including $617 \mathrm{TNBC}$, were acquired, normalized
\end{abstract}

Electronic supplementary material The online version of this article (doi:10.1007/s10549-017-4195-7) contains supplementary material, which is available to authorized users.

Ling-Ming Tseng

lmtseng@vghtpe.gov.tw

Jen-Hwey Chiu

chiujh@ym.edu.tw

Chun-Yu Liu

cyliu3@vghtpe.gov.tw

Yi-Fang Tsai

yftsai@vghtpe.gov.tw

Yun-Lin Wang

resentis@gmail.com

Chu-Wen Yang

ycw6861@scu.edu.tw

Yi-Ming Shyr

ymshyr@vghtpe.gov.tw

1 Comprehensive Breast Health Center \& Division of General Surgery, Department of Surgery, Taipei Veterans General Hospital, No. 201, Sec. II, Shih-pei Road, Taipei 112,

Taiwan, ROC and cluster analyzed. Then, using TNBC cell lines of different subtypes, namely, MDA-MB-468 (basal-like1, BL1), MDA-MB-231 (mesenchymal stem like, MSL), BT549 (mesenchymal, M), MDA-MB-453 (luminal androgen receptor, LAR), and DU4475 (immunomodulatory, IM), real-time PCR in triplicate for 47 genes signatures were performed to validate the specificity of these subtypes. Results The results showed that the percentage of TNBC subtypes in non-Asian women, namely, BL1, BL2, IM, M, MSL, and LAR was $13.56,8.91,16.80,20.45,8.30$, and $11.13 \%$, respectively. When data from Taiwanese were normalized and clustered, five TNBC subtypes, namely, BL (8.94\%), IM (13.82\%), M (22.76\%), MSL (30.89\%), and LAR (23.58\%), were classified. Real-time PCR validated the specificity of these subtypes. Besides, the

2 Department of Surgery, School of Medicine, National YangMing University, Taipei, Taiwan, ROC

3 Institute of Traditional Medicine, School of Medicine, National Yang-Ming University, Taipei, Taiwan, ROC

4 Division of General Surgery, Department of Surgery, ChengHsin General Hospital, Taipei, Taiwan, ROC

5 Division of Medical Oncology, Department of Oncology, Taipei Veterans General Hospital, Taipei, Taiwan, ROC

6 School of Medicine, Institute of Clinical Medicine, National Yang-Ming University, Taipei, Taiwan, ROC

7 Department of Microbiology, Soochow University, Taipei, Taiwan, ROC 
presence of interaction between IM- and MSL-subtypes suggests the involvement of tumor microenvironment in TNBC subtype classification.

Conclusion Our data suggested that there exist different presentations between non-Asian and Taiwanese TNBC subtypes, which provides important information when selection of therapeutic targets or designs for clinical trials for TNBC patients.

Keywords Triple negative · Breast cancer - Subtype · Gene expression · Precision medicine

$\begin{array}{ll}\text { Abbreviations } \\ \text { BL } & \text { Basal-like } \\ \text { CDF } & \text { Cumulative Distribution Function } \\ \text { ER } & \text { Estrogen receptor } \\ \text { GE } & \text { Gene expression } \\ \text { HER2 } & \text { Human epidermal growth receptor } 2 \\ \text { IM } & \text { Immune-modulatory } \\ \text { M } & \text { Mesenchymal } \\ \text { MSL } & \text { Mesenchymal stem cell like } \\ \text { LAR } & \text { Luminal androgen receptor } \\ \text { PR } & \text { Progenstin receptor } \\ \text { TNBC } & \text { Triple-negative breast cancer }\end{array}$

\section{Introduction}

Precision medicine has become an important emerging approach to the diagnosis, treatment, and prevention of disease, especially cancers; it takes into account the individual variability of each person in terms of genes, environment, and lifestyle. Breast cancer is the most common malignancy in women $[1,2]$. Owing to tumor heterogeneity caused by cell phenotype diversity, different approaches to treatment and prognosis have been shown to be highly correlated with the intrinsic subtypes of the breast cancer [3]. Triple-negative breast cancer [TNBC, ER(-), PR(-), HER2(-)], which accounts for about $15 \%$ of breast cancers worldwide, is characterized by aggressive tumor behavior and a strong resistance to ant hormone treatment, chemotherapy, and targeted therapy [4-6].

Previously, using whole-genome (genome wide) analysis, including gene expression analysis (gene expression profiling), various TNBC molecular subtypes have been further identified. For example, six specific subtypes, namely, basallike1 (BL1), basallike2 (BL2), mesenchymal (M), mesenchymal stem like (MSL), immune response (M), and luminal androgen receptor positive (LAR) were first described by Lehmann et al. [7]. Since then, more investigations have targeted TNBC tumor heterogeneity using gene ontology [8-10], therapeutic targets [11, 12], and using mRNA or long noncoding RNAs (lncRNAs) as diagnostic criteria [13]. Although the six subtype classification has been refined recently [14, 15], the variation in molecular classification of TNBC across various different populations remains to be elucidated.

Accumulating evidence has shown that social economic, epidemiological, and genetic factors all play roles in tumor behavior, cancer subtype, and the prognosis of patients among different racial/ethnic groups [16-18]. For example, women of African heritage, compared to women of Caucasian heritage, have a higher rate of TNBC and a lower rate of receptor $(+) / \operatorname{HER} 2(-)$ breast cancers after the age of 35 years [19]. Furthermore, a high prevalence and poorer clinical outcomes have been observed among AfricanAmerican women with TNBC than among women of European descent [20, 21]. There is consensus that genomewide studies, such as gene expression profile analysis, provide multi-gene signatures that are closely linked to TNBC carcinogenesis [22, 23]. Previous studies have demonstrated a significant association between the PTEN mutation, a high Ki67 index and the CD44 ${ }^{+} / \mathrm{CD} 24$ phenotype among African-American women with TNBC [24]. In addition to the above findings, it has also been noted that there are frequently variations in the EGFR-activating mutations found in TNBCs among East Asians patients and this is not true for European patients [25]. In the context of these findings, controversy exists regarding the amount of variations that occurs in genomic profiles between different ethnic populations [26]. Therefore, the aim of the present study was to compare the molecular subtypes of triple-negative breast cancers (TNBCs) between Taiwanese female patients and nonunion female patients.

\section{Methods}

\section{Subjects}

Under the approval of the Institutional Review Board (\# 201310020BC) of Taipei Veterans General Hospital, Taiwan, ROC, a total of 57 patients between June 2013 and September 2015 with TNBC [ER(-), PR(-), HER2(-)] were identified by immunohistochemical analysis of their pathological specimens. Total RNA was extracted from these TNBC tissue samples, and the RNA samples were used to conduct oligonucleotide microarray analysis by the Genome Research Center, National Yang-Ming University [27].

\section{Data set collection and TNBC identification by bimodal filtering}

GE profiles from fourteen publicly available breast cancer microarray datasets, including twelve nonunion and two 
Table 1 Triple-negative breast cancer (TNBC) distribution in publicly available data sets

\begin{tabular}{|c|c|c|c|c|c|c|}
\hline \multicolumn{3}{|l|}{ Non-Asian } & \multirow[t]{2}{*}{ Country } & \multicolumn{3}{|l|}{ Taiwanese } \\
\hline GEO accession & BC case & TNBC & & GEO accession & $\mathrm{BC}$ case & TNBC \\
\hline GSE12276 & 204 & 67 & Netherlands & GSE20685 & 327 & 57 \\
\hline GSE14017 & 29 & 13 & USA & GSE48390 & 81 & 16 \\
\hline GSE17907 & 51 & 1 & France & GSE95700 (VGH) & 57 & 50 \\
\hline GSE18864 & 84 & 53 & Denmark & & & \\
\hline GSE19615 & 115 & 35 & USA & & & \\
\hline GSE19697 & 24 & 24 & USA & & & \\
\hline GSE20711 & 88 & 24 & Canada & & & \\
\hline GSE21653 & 266 & 91 & France & & & \\
\hline GSE31448 & 353 & 131 & France & & & \\
\hline GSE42568 & 104 & 32 & Ireland & & & \\
\hline GSE43502 & 25 & 19 & USA & & & \\
\hline GSE58812 & 107 & 96 & France & & & \\
\hline Sum & 1450 & 494 & & Sum & 465 & 123 \\
\hline
\end{tabular}

$B C$ breast cancer, $T N B C$ triple-negative breast cancer, $V G H$ Veterans General Hospital
Taiwanese datasets (Sun Yat-Sen Cancer Center and Cathy hospital) (GEO, http://www.ncbi.nlm.nih.gov/gds; Array Express, http://www.ebi.ac.uk/microarrayas/ae/) were compiled and these were added to our dataset (GSE95700) (Supplementary Reference 1). In total, 1915 human breast cancer samples were included and among these samples a total of 617 TNBCs were identified (Table 1). The GE raw values for each of the datasets were normalized independently using the RMA procedure. The Affymetrix probes used for ER, PR, and HER2 were 205225_at, 208305_at, and 216836_s_at, respectively. A two-component Gaussian mixture distribution model was used to analyze the empirical expression distributions of ER, PR, and HER2 and the default parameters were estimated by maximum likelihood optimization using R statistical software (https:// www.rproject.org/). After the posterior probability of a negative expression state for ER, PR, and HER2 had been estimated, a sample was defined as having negative expression if the posterior probability was less than 0.5 . This process was followed by bimodal filtering to remove

Table 2 Gene list for validation of Taiwanese TNBC subtype

\begin{tabular}{|c|c|c|c|c|c|c|c|c|c|}
\hline \multicolumn{2}{|c|}{ Subtype 1 (IM) } & \multicolumn{2}{|c|}{ Subtype 2 (MSL) } & \multicolumn{2}{|c|}{ Subtype 3 (M) } & \multicolumn{2}{|c|}{ Subtype 4 (LAR) } & \multicolumn{2}{|c|}{ Subtype 5 (BL) } \\
\hline Probe & $\begin{array}{l}\text { Gene } \\
\text { symbol }\end{array}$ & Probe & $\begin{array}{l}\text { Gene } \\
\text { symbol }\end{array}$ & Probe & $\begin{array}{l}\text { Gene } \\
\text { symbol }\end{array}$ & Probe & $\begin{array}{l}\text { Gene } \\
\text { symbol }\end{array}$ & Probe & $\begin{array}{l}\text { Gene } \\
\text { symbol }\end{array}$ \\
\hline 232362_at ${ }^{\mathrm{a}}$ & CCDC18 & 227427_at & ARHGEF25 & 201268_at & $\begin{array}{l}\text { NME1- } \\
\text { NME2 }\end{array}$ & 218211_s_at & MLPH & 219787_s_at & ECT2 \\
\hline 206486_at & LAG3 & 206485_at & CD5 & 213801_x_at & RPSA & 215465_s_at & ABCA12 & 231984_at & MTAP \\
\hline 207634_at & PDCD1 & 217190_x_at & ESR1 & 200023_s_at & EIF3F & 232914_s_at & SYTL2 & 229538_s_at & IQGAP3 \\
\hline 223834_at & $\mathrm{CD} 274$ & 211233_x_at & ESR1 & 215157_x_at & PABPC1 & 212510_at & GPD1L & 208165_s_at & PRSS16 \\
\hline 220049_s_at & PDCD1LG2 & 215104_at & NRIP2 & 228256_s_at & EPB41L4A & 227733_at & TMEM63C & 226189_at & ITGB8 \\
\hline 222835_at & THSD4 & 229377_at & GRTP1 & 205990_s_at & WNT5A & 235020_at & TAF4B & 212998_x_at & $\begin{array}{l}\text { HLA- } \\
\text { DQB1 }\end{array}$ \\
\hline 228708_at & RAB27B & 244264_at & KLRG2 & 226192_at & $\mathrm{AR}$ & 209138_x_at & IGLC1 & 215536_at & $\begin{array}{l}\text { HLA- } \\
\text { DQB2 }\end{array}$ \\
\hline 209505_at & NR2F1 & 232179_at & LOC158863 & 204014_at & DUSP4 & 225973_at & TAP2 & 204149_s_at & GSTM4 \\
\hline \multirow[t]{2}{*}{ 226553_at } & TMPRSS2 & 236390_at & SLX4IP & 203963_at & CA12 & 223307_at & CDCA3 & 214123_s_at & $\begin{array}{c}\text { NOP14- } \\
\text { AS1 }\end{array}$ \\
\hline & & 213823_at & HOXA11 & & & 232001_at & $\begin{array}{c}\text { PRKCQ- } \\
\text { AS1 }\end{array}$ & & \\
\hline
\end{tabular}

a Gene probes were derived from Affi-matrix microarray GE

$I M$ immumodulatory, $M S L$ mesenchymal stem like, $M$ mesenchymal, $L A R$ luminal androgen receptor, $B L$ basal-like 
Fig. 1 Protocol for the acquisition and analysis of the gene expression datasets. GEO Datasets for nonunion (12 groups, $n=1450$ ) and Taiwanese ( 3 groups, $n=465$ ) female breast cancer samples, including 617 triple-negative breast cancer (TNBC) samples, were acquired, normalized, and cluster analyzed (a). TNBC was identified by bimodal filtering (b) and was demonstrated in (c) a

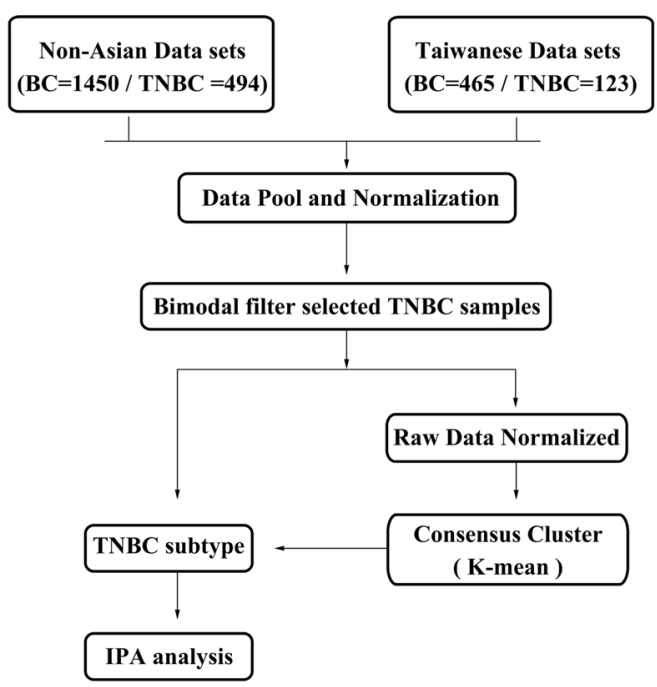

Bimodal Filter

b

PR (208305_at)

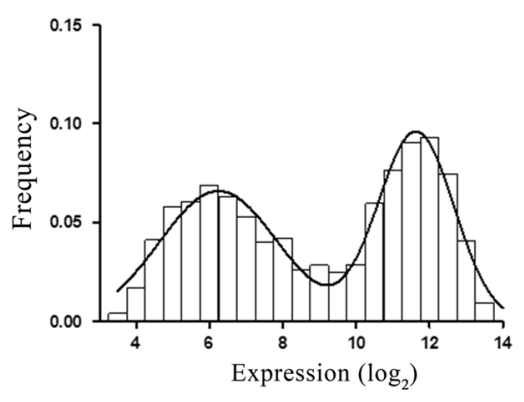

HER2 (216836_s_at)

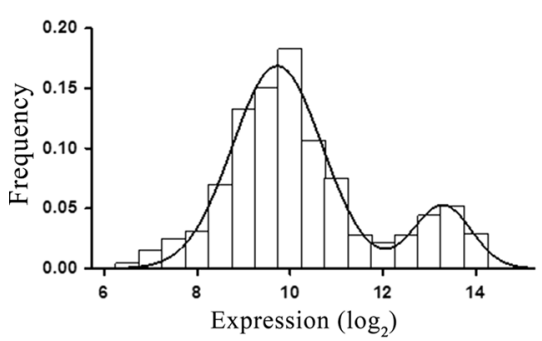

C

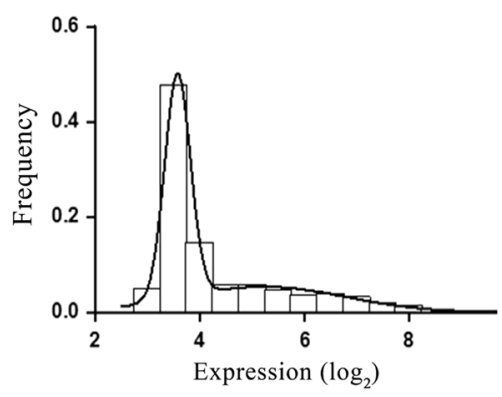

\begin{tabular}{|c|c|}
\hline Gene symbol & cutoff \\
\hline ESR1 & 9.207865 \\
\hline PR & 4.432565 \\
\hline HER2 & 12.04873 \\
\hline
\end{tabular}

\begin{tabular}{lll}
$\mathrm{ER}^{+}$ & $\mathrm{PR}^{+} \quad \mathrm{HER} 2^{+}$ \\
\hline
\end{tabular}

\section{TNBC GE}

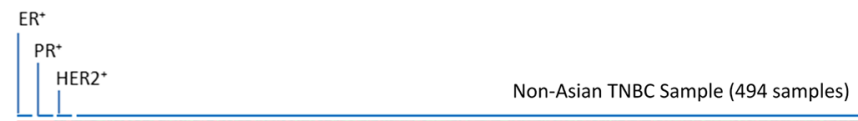

$-4$ 


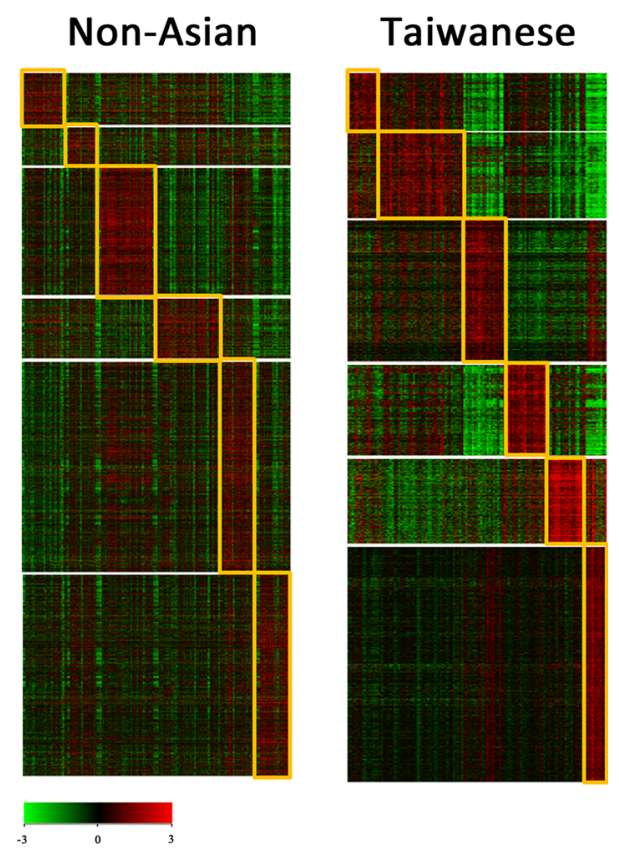

Fig. 2 Heat maps of the clustered triple-negative breast cancer (TNBC) subtype for nonunion and Taiwanese women. The published gene lists of the six subtypes of TNBC were imported and used for the clustering of our compiled dataset, which consisted of a nonunion group (left panel) and a Taiwanese group (right panel) TNBC

all ER/PR/HER2 positive tumors. The remaining TNBC tumors were then normalized along with positive controls for ER, PR, and HER2. Only samples that displayed a marked reduction in expression based on the above criteria compared to the positive controls were classified as TNBC $(n=617)$.

\section{Identification of TNBC subtypes}

Previously, six distinct TNBC molecular subtypes were proposed by Lehmann et al. [7] and these were basallike1 (BL1), basallike2 (BL2), mesenchymal (M), mesenchymal stem-like (MSL), immune response (M), and luminal androgen receptor positive (LAR). Accordingly, using the published six type gene lists, we clustered and replotted the six types of heat map using our compiled complete dataset. In addition to background correction, the MAS5 procedure was applied to the Taiwanese data and then consensus clustering and $k$-means clustering were used to determine the optimal number of stable TNBC subtypes. Cluster robustness was assessed by consensus clustering using agglomerative k-means clustering using the average linkage for the 123 TNBC profiles based on the most differentially expressed genes (SD $>0.9 ; n=5463$ genes). The optimal number of clusters was determined from the Consensus Cumulative Distribution Function (CDF), which plotted the corresponding empirical cumulative distribution; this was defined over the range [0,1], and calculated based on the proportional increase in the area under the CDF curve. Following this, the number of clusters was decided when any further increase in cluster number $(k)$ did not lead to a corresponding marked increase in the CDF area. Principal component analysis (PCA) and heat maps were generated using GeneSpring software (GeneSpring GX 11.5; Agilent Technologies, Inc., Santa Clara, CA, USA) and further pathway analysis was carried out using Ingenuity Pathway Analysis software [27] (IPA; Qiagen, Redwood City, CA, USA).

\section{Gene selection specific to each TNBC subtype}

After consensus clustering and k-means clustering of the Taiwanese data, the TNBC subtypes were determined. The genes specific to each TNBC subtype were defined as followings: (1) the strongest probe with a fold change (ratio), $>1.75$ (upregulation) or $<0.5$ (downregulation), compared with the other subtypes; (2) the percentage of the sample with a GE difference $>0$ (sample GE - mean GE of other subtypes) of $>80 \%$; and a $p$ value $<10^{4}$ ( $t$ test: specific subtype versus other subtypes).

\section{Cell line and reagents}

Under the approval of Institutional Review Board (\# 201606012BC) of Taipei Veterans General Hospital, Taiwan, ROC, the human triple-negative breast cancer cell lines MDA-MB-468 (BL1), MDA-MB-231 (MSL), BT549 (M), MDA-MB-453 (LAR), and DU4475 (IM) were obtained from the American Type Culture Collection (ATCC, Manassas, VA, USA), and these were then maintained in specific culture medium, namely F12 MEM (No. 12400024, Gibco, NY, USA), RPMI, as appropriate; the media were supplemented with $10 \%$ FBS, $2 \mathrm{mM}$ L glutamine and penicillin/streptomycin, and the cells were cultured at $37{ }^{\circ} \mathrm{C}$ in a humidified atmosphere containing $5 \% \mathrm{CO}_{2}$. Cells that were from three passages to ten passages were used.

\section{Total RNA extraction and reverse transcription PCR}

Total RNA was isolated using the modified single step guanidinium thiocyanate method [28] (TRI REAGENT, T9424, Sigma Chem. Co., St. Louis, MO, USA). After the cells from the five different subtypes, namely, MDA-MB468 (BL1), MDA-MB-231 (MSL), BT-549 (M), MDAMB-453 (LAR), and DU4475 (IM) had been grown up and total RNAs extracted, complementary DNA (cDNA) was created using a First Strand cDNA Synthesis Kit (Invitrogen, CA, USA). TaqMan ${ }^{\circledR}$ Gene Expression Assays were 


\section{Clustering for TNBC subtype in Taiwanese}

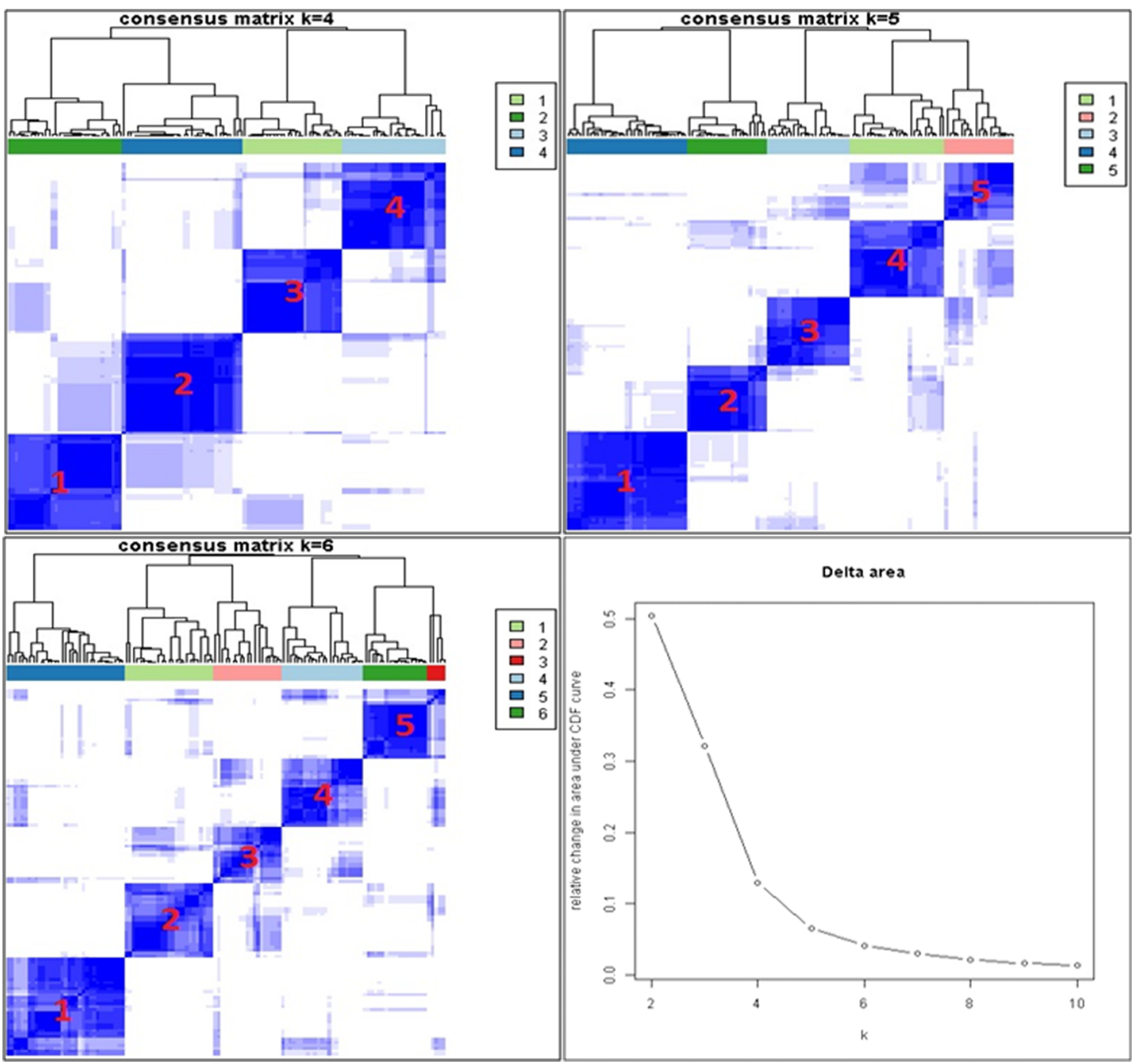

Fig. 3 Cluster analysis of the triple-negative breast cancer (TNBC) subtype for Taiwanese women. After background correction of the Taiwanese data, consensus clustering and k-means clustering were

used to validate the differential expression at the mRNA level of the various identified genes sets that had been selected from consensus clustering results (Table 2). The TaqMan system was supported by a well-established primer database that reduces significantly the experimental failure due to inappropriate primer design.

Any possible contamination of the various PCR components was excluded by performing a PCR reaction with these components in the absence of the RT product for each set of experiments (contemplate control, NTC). For the statistical comparisons, the relative expression level of the mRNA of each specific gene was normalized against the used to determine the optimal number of TNBC subtypes. The optimal number of clusters was determined from the Consensus Cumulative Distribution Function (CDF)

amount of GAPD mRNA in the same RNA extract. All samples were analyzed in triplicate.

\section{Statistic analysis}

Data are expressed as mean \pm SEM. Differences between groups were identified by repeatedly measured one-way ANOVA, followed by Dunnet's post hoc test. Differences between different groups were identified by Mann-Whitney $U$ test for nonparametric analysis or the Student's $t$ test. A $p$ value of $<0.05$ is considered statistically significant. 
Fig. 4 The triple-negative breast cancer (TNBC) subtypes for TNBC from Taiwanese women. The heat map shows five stable TNBC subtypes (a). The genes specific to each subtype are 274227458_at (CD 274 or $P D L 1$ ) for IM, 205225_at for MSL,

200091_s_at for M, 226192_at (androgen receptor) for LAR, and 229538_s_at (IQGAP3) for BL (b) a

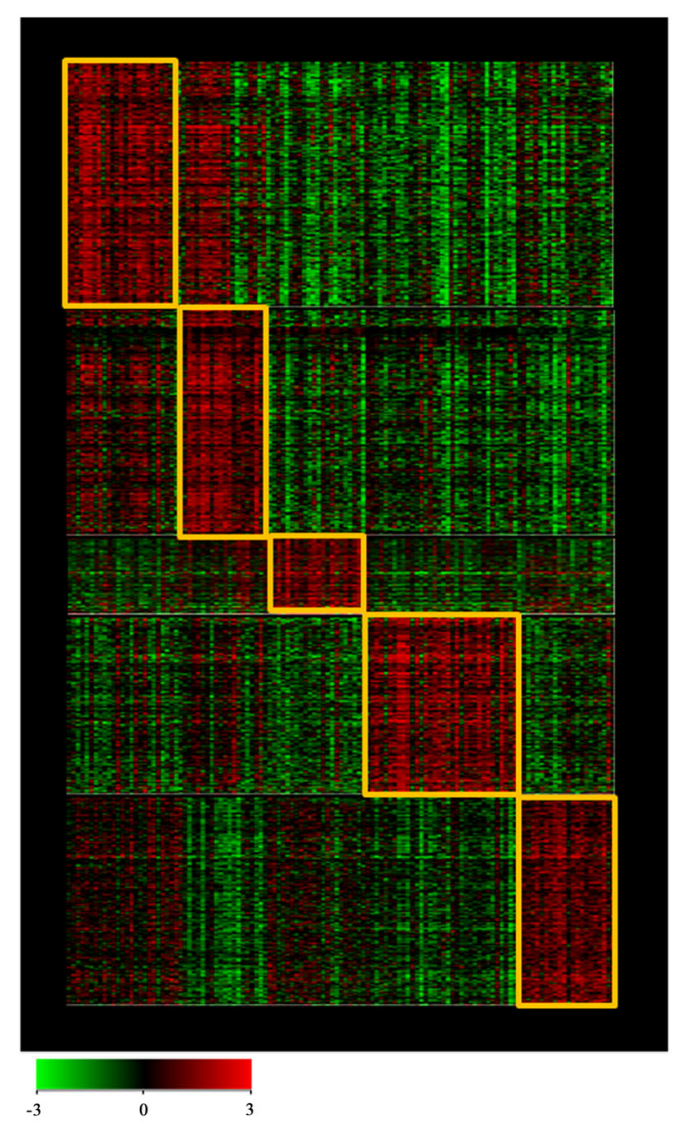

b
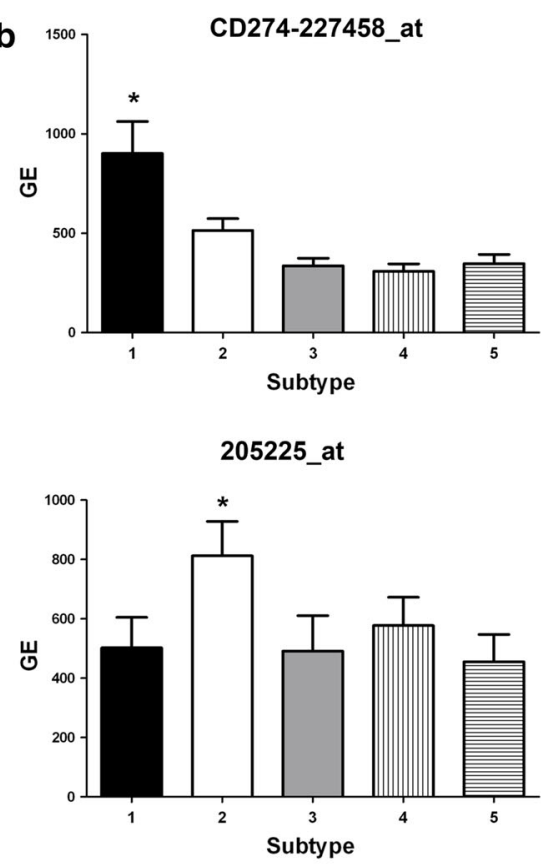

RPS25-200091_s_at

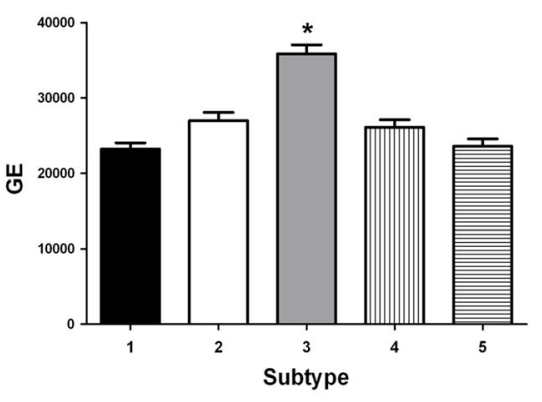

226192_at

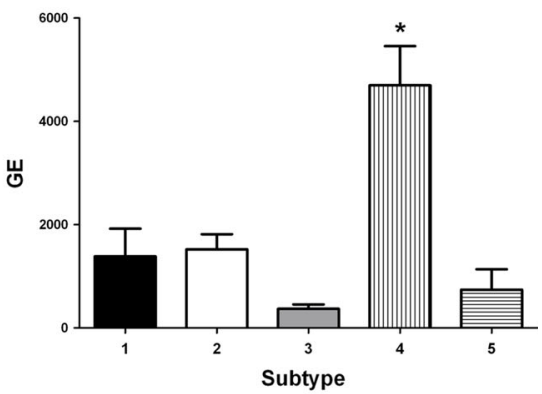

IQGAP3-229538_s_at

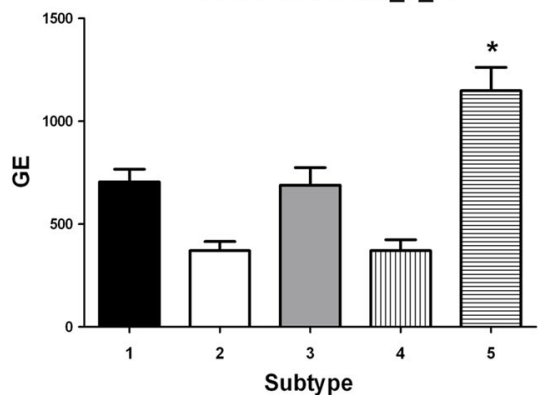


Table 3 Correlation of subtype-specific genes between Taiwanese's and Lehmann's genes

\begin{tabular}{lccccc}
\hline Lehmann's subtypes & Present study & & & \\
\cline { 2 - 6 } & Subtype1 (IM) & Subtype2 (MSL) & Subtype3 (M) & Subtype4 (LAR) & Subtype5 (BL) \\
\hline BL1 & $19.23^{\mathrm{a}}$ & 0.00 & 8.70 & 0.00 & 50.00 \\
BL2 & 0.00 & 5.26 & 8.70 & 3.03 & 4.55 \\
IM & 53.85 & 26.32 & 13.04 & 0.00 & 0.00 \\
M & 19.23 & 5.26 & 34.78 & 9.09 & 13.64 \\
MSL & 0.00 & 52.63 & 0.00 & 24.24 & 4.55 \\
LAR & 3.85 & 10.53 & 63.64 & 4.55 \\
\hline
\end{tabular}

a Data were presented as percentage $(\%)$

$I M$ immumodulatory, $M S L$ mesenchymal stem like, $M$ mesenchymal, $L A R$ luminal androgen receptor, $B L$ basal-like

\section{Results}

\section{Dataset collection and TNBC identification by bimodal filtering}

From June 2013 to September 2015, 57 patients whose tumor samples were screened as TNBC by immunohistochemistry (ER $<1 \%$, PR $<1 \%$, HER2, not amplified) were identified at Taipei Veterans General Hospital. These tumor samples were sent for microarray analysis. Next, two Taiwanese $(n=408)$ and twelve nonunion datasets $(n=1450)$ were downloaded from the public domain. Thus, a total of 1915 human breast cancer samples, including ours $(n=57)$, were available for expression analysis. The gene expression information generated from Affymetrix microarrays were then normalized independently using RMA procedures (Fig. 1a and Supplementary Reference 1).

The gene expression distributions of ER, PR, and HER2 for the TNBC samples were validated by two-component Gaussian distribution, and the cutoff point was estimated by maximum likelihood optimization using the optimize function (R statistical software) (Fig. 1b). This resulted in a heat map showing the TNBC tumors normalized along with positive controls for ER, PR, and HER2 (Fig. 1c). Finally, the TNBCs identified as true TNBCs $(n=617)$ were enrolled into the compiled dataset.

\section{The GE TNBC subtype samples of nonunion and Taiwanese women clustered in terms of the published 6-subtype gene lists}

Since TNBC subtyping has been suggested as a useful approach, we acquired the published gene lists of the 6-subtype of TNBC and used these for clustering of our compiled dataset, which included nonunion (Fig. 2, left panel) and Taiwanese (Fig. 2, right panel) women. The results showed that the percentages of TNBC subtypes in nonunion women, namely, BL1, BL2, IM, M, MSL, and
LAR were $13.56,8.91,16.80,20.45,8.30$, and $11.13 \%$, respectively, while those in Taiwanese women was 14.63 , $4.07,17.89,16.26,17.89$, and $20.33 \%$, respectively.

When the two groups of women are compared, there exist some discrepancies between nonunion and Taiwanese women in terms of TNBC subtypes. To address this, background correction for the Taiwanese data was performed and consensus clustering and $k$-means clustering were used to determine the optimal number of TNBC subtypes for Taiwanese (Fig. 3). The results showed that five stable subtypes were obtained based on the Taiwanese TNBC data (Fig. 4a). These were IM (13.82\%), MSL (30.89\%), M (22.76\%), LAR (23.58\%) and BL (8.94\%). The genes specific to each subtype were 274227458_at (CD 274 or PDL1) for IM, 205225_at for MSL, 200091_s_at for M, 226192_at (androgen receptor) for LAR, and 229538_s_at (IQGAP3) for BL (Fig. 4b). The genes specific to each TNBC subtype having been identified (Supplementary Reference 2) and correlated with the Lehmann et al. genes (Table 3) were analyzed using ingenuity pathway analysis (IPA); furthermore, their top canonic pathways, their upstream regulators, their top disease and their biofunctions were also analyzed. The results are summarized in Tables 4 and 5 .

\section{Model identification using representative genes in human TNBC cell lines}

Using the gene lists selected from the clustering results (Supplementary Reference 2), which were identified as specific to each subtype, real-time PCR was carried targeting a 47 gene signature (Table 2) using customized chip. This analysis was carried out on five human TNBC cell lines, namely, MDA-MB-468 (BL1), MDA-MB-231 (MSL), BT-549 (M), MDA-MB-453 (LAR), and DU4475 (IM).

Using DU4475 (IM) as the reference line, significant downregulation of THSD4, ECT2, RAB27B, and ITGB8 was found (Fig. 5a), together with significant upregulation 
Table 4 Ingenuity pathway analysis for up-regulated genes in TNBC subtypes

Name $\quad p$-value

Subtype 01 (immunomodulatory)

Top canonical pathways

CD28 signaling in $\mathrm{T}$ helper cells

$7.02 \mathrm{E}-17$

iCOS-iCOSL signaling in $\mathrm{T}$ helper cells

$1.24 \mathrm{E}-16$

Natural killer cell signaling

$9.45 \mathrm{E}-15$

Role of NFAT in regulation of the immune response $2.08 \mathrm{E}-13$

$\mathrm{T}$ cell receptor signaling

$3.69 \mathrm{E}-13$

Top upstream regulators

E2F4/IRF7/IRF1/E2F1/ESR1

Top diseases and bio functions

Cancer/organismal injury and abnormalities/gastrointestinal disease/ infectious diseases/hematological disease

Subtype 02 (mesenchymal stem like)

Top canonical pathways

EIF2 signaling

iCOS-iCOSL signaling in $\mathrm{T}$ helper cells

1.19E-17

Hepatic fibrosis/hepatic stellate cell activation

$1.15 \mathrm{E}-14$

Crosstalk between dendritic cells and natural killer

$1.27 \mathrm{E}-14$ cells

$3.20 \mathrm{E}-14$

Tec kinase signaling

$2.89 \mathrm{E}-12$

Top upstream regulators

CREBBP/MYCN/EP300/ID2/BCL6

Top diseases and bio functions

Cancer/organismal injury and abnormalities/inflammatory

response/connective tissue disorders/skeletal and muscular disorders

Subtype 03 (mesenchymal)

Top canonical pathways

$$
\text { EIF2 signaling }
$$

$3.18 \mathrm{E}-69$

Regulation of eIF4 and p70S6K signaling

$2.92 \mathrm{E}-23$

Oxidative phosphorylation

$4.38 \mathrm{E}-18$

mTOR signaling

$1.45 \mathrm{E}-16$

Mitochondrial dysfunction

$5.81 \mathrm{E}-14$

Top upstream regulators

\section{MYCN/MYC/HNF4A/DOT1L/HSF1}

Top diseases and bio functions

Cardiovascular disease/developmental disorder/hereditary disorder/ organismal injury and abnormalities

Subtype 04 (luminal androgen receptor)

Top canonical pathways

NRF2-mediated oxidative stress response

Xenobiotic metabolism signaling

$8.86 \mathrm{E}-08$

$3.00 \mathrm{E}-06$

$1.64 \mathrm{E}-05$

LPS/IL-1 mediated Inhibition of RXR function

$5.69 \mathrm{E}-05$

HIPPO signaling

Clathrin-mediated endocytosis signaling

$7.13 \mathrm{E}-05$

Top upstream regulators

\section{ESR1/HNF4A/TP53/PGR/ESR2}

Top diseases and bio functions

Cancer/organismal injury and abnormalities/gastrointestinal disease/ hepatic system disease/developmental disorder
Table 4 continued

\begin{tabular}{ll}
\hline Name & $p$-value \\
\hline Subtype 05 (basal-like) & \\
Top canonical pathways & \\
Role of BRCA1 in DNA damage response & $2.92 \mathrm{E}-15$ \\
Hereditary breast cancer signaling & $4.71 \mathrm{E}-14$ \\
Cell cycle: G2/M DNA damage checkpoint regulation & $2.32 \mathrm{E}-13$ \\
Role of CHK proteins in cell cycle checkpoint control & $4.39 \mathrm{E}-13$ \\
Mitotic roles of polo-like kinase & $2.82 \mathrm{E}-12$
\end{tabular}

Top upstream regulators

E2F4/HNF4A/NUPR1/E2F1/ESR1

Top diseases and bio functions

Cancer/organismal injury and abnormalities/gastrointestinal disease/ infectious diseases/hepatic system disease

Table 5 Ingenuity pathway analysis for down-regulated genes in TNBC subtypes

\begin{tabular}{ll}
\hline Name & $p$-value \\
\hline Subtype 01 (immunomodulatory) & \\
Top canonical pathways & \\
EIF2 signaling & $6.04 \mathrm{E}-25$ \\
Regulation of eIF4 and p70S6K signaling & $2.40 \mathrm{E}-11$ \\
mTOR signaling & $9.41 \mathrm{E}-10$ \\
Mitochondrial dysfunction & $3.25 \mathrm{E}-08$ \\
Tight junction signaling & $2.08 \mathrm{E}-07$
\end{tabular}

Top upstream regulators

\section{MYCN/ESR1/HNF4A/CREB1/PGR}

Top diseases and bio functions

Cancer/organismal injury and abnormalities/neurological disease/ psychological disorders/gastrointestinal disease

Subtype 02 (mesenchymal stem like)

Top canonical pathways

Protein ubiquitination pathway $\quad 4.08 \mathrm{E}-20$

Role of CHK proteins in cell cycle checkpoint control $1.19 \mathrm{E}-13$

Mitotic roles of polo-like kinase $\quad 1.45 \mathrm{E}-12$

Hypoxia signaling in the cardiovascular system $1.14 \mathrm{E}-10$

Role of BRCA1 in DNA damage response 1.29E-09

Top upstream regulators

HNF4A/E2F4/ESR1/TP53/NUPR1

Top diseases and bio functions

Cancer/organismal injury and abnormalities/gastrointestinal disease/ infectious diseases/hepatic system disease

Subtype 03 (mesenchymal)

Top canonical pathways

B cell receptor signaling

$2.16 \mathrm{E}-18$

Leukocyte extravasation signaling

$7.44 \mathrm{E}-16$

Integrin signaling

$5.48 \mathrm{E}-15$

Molecular mechanisms of cancer

$1.05 \mathrm{E}-14$

Hepatic fibrosis/hepatic stellate cell activation $\quad 4.83 \mathrm{E}-12$ 
Table 5 continued

\begin{tabular}{|c|c|}
\hline Name & $p$-value \\
\hline \multicolumn{2}{|l|}{ Top upstream regulators } \\
\hline \multicolumn{2}{|l|}{ ESR1/HNF4A/TP53/ERG/NR3C1 } \\
\hline \multicolumn{2}{|l|}{ Top diseases and bio functions } \\
\hline \multicolumn{2}{|c|}{$\begin{array}{l}\text { Cancer/organismal injury and abnormalities/gastrointestinal disease } \\
\text { hepatic system disease/reproductive system disease }\end{array}$} \\
\hline \multicolumn{2}{|l|}{ Subtype 04 (luminal androgen receptor) } \\
\hline \multicolumn{2}{|l|}{ Top canonical pathways } \\
\hline Role of BRCA1 in DNA damage response & $3.35 \mathrm{E}-14$ \\
\hline Molecular mechanisms of cancer & $4.43 \mathrm{E}-11$ \\
\hline Hereditary breast cancer signaling & $1.03 \mathrm{E}-10$ \\
\hline $\begin{array}{l}\text { Crosstalk between dendritic cells and natural killer } \\
\text { cells }\end{array}$ & $2.98 \mathrm{E}-10$ \\
\hline Natural killer cell signaling & $6.01 \mathrm{E}-10$ \\
\hline \multicolumn{2}{|l|}{ Top upstream regulators } \\
\hline \multicolumn{2}{|l|}{ E2F4/IRF7/E2F1/CDKN2A/IRF1 } \\
\hline \multicolumn{2}{|l|}{ Top diseases and bio functions } \\
\hline \multicolumn{2}{|c|}{$\begin{array}{l}\text { Cancer/organismal injury and abnormalities/gastrointestinal disease } \\
\text { infectious diseases/hematological disease }\end{array}$} \\
\hline \multicolumn{2}{|l|}{ Subtype 05 (basal-like) } \\
\hline \multicolumn{2}{|l|}{ Top canonical pathways } \\
\hline EIF2 signaling & $3.99 \mathrm{E}-20$ \\
\hline Hepatic fibrosis/hepatic stellate cell activation & $1.52 \mathrm{E}-17$ \\
\hline $\begin{array}{l}\text { Crosstalk between dendritic cells and natural killer } \\
\text { cells }\end{array}$ & $1.58 \mathrm{E}-12$ \\
\hline Primary immunodeficiency signaling & $1.13 \mathrm{E}-09$ \\
\hline LPS/IL-1 mediated inhibition of RXR function & $1.91 \mathrm{E}-09$ \\
\hline \multicolumn{2}{|l|}{ Top upstream regulators } \\
\hline \multicolumn{2}{|l|}{ MYCN/CREBBP/EP300/SMARCA4/CTNNB1 } \\
\hline \multicolumn{2}{|l|}{ Top diseases and bio functions } \\
\hline $\begin{array}{l}\text { Cancer/organismal injury and abnormalities/dermatol } \\
\text { and conditions/connective tissue disorders/inflammat }\end{array}$ & $\begin{array}{l}\text { al disease } \\
\text { disease }\end{array}$ \\
\hline
\end{tabular}

of PDCD1 (PD1), CD274 (PDL1) (except MDAMB231), and PDCD1LG2 (PDL2) (Fig. 5b), in DU4475 compared to the other cell lines MDA-MB-468 (BL1), MDA-MB-231 (MSL), BT-549 (M), and MDA-MB-453 (LAR). Using MDA-MB-231 (MSL) as the reference line, significant upregulation of DUSP4, together with significant downregulation of $C C D C 18$ and GRTP1 (Fig. 5c) were found in MDA-MB-231 compared to the other cell lines. Using BT$549(\mathrm{M})$ as the reference line, significant upregulation of CDCA3 and MATP in BT-549 (Fig. 5d) was found compared to the other cell lines. However, in addition these findings for BT-549, it needs to be noted that there was significant upregulation of DUSP4 in MDA-MB-231 (MSL) and of $A R$ in MDA-MB-453 (LAR) compared to BT-549 (M) (Fig. 5d). When using MDA-MB-453 (LAR) as the reference line, significant upregulation of $A R$,
Fig. 5 Model identification using representative genes in human triple-negative breast cancer (TNBC) cell lines. Using the DU4475 (IM) as the reference line, there was significant downregulation of THSD4, ECT2, RAB27B, and ITGB8 (a) together with significant upregulation of PDCD1 (PD1), CD274 (PDL1) (except MDAMB231), and PDCD1LG2 (PDL2) (b) compared to the other cell lines).Using the MDA-MB-231 (MSL) (c) as the reference line, there was significant upregulation of DUSP4 together with significant downregulation of CCDC18 and GRTP1 compared to other cell lines. Using the BT-549 (M) (d) as the reference line, there was significant upregulation of $C D C A 3$ and MATP in this line, compared to other cell lines and there was significant upregulation of DUSP4 in MDA-MB231 (MSL) and AR in MDA-MB-453 (LAR), compared to the BT549 (M) line. Using the MDA-MB-453 (LAR) as reference line (e), there was significant upregulation of $A R, A B C A 12, I Q G A P 3$, and $K L R G 2$ in this line, compared to other cell lines. Using the MDAMB-468 (BL1) as the reference line (f), there was significant upregulation of $T P G B 8, P A B P C 1$, and $W N T 5 A$ in this line, compared to other cell lines

ABCA12, IGQAP3, and KLRG2 in MDA-MB-453 (Fig. 5e) was found. Finally, when using MDA-MB-468 (BL1) as the reference line, significant upregulation of ITGB8, $P A B P C 1$, and WNT5A in MDA-MB-468 (Fig. 5f) was found.

\section{Discussion}

Breast cancer raises important health problem worldwide. Even after considering the many therapies for the various subtypes of breast cancer, treatment of triple-negative breast cancer (TNBC) remains a challenging issue. The heterogeneity of TNBC tumors contributes to their poor response to chemotherapy, and this had led to the development of TNBC subtyping. In this study, we compiled GE profiles from publically available breast cancer microarray datasets that included both nonunion and Taiwanese populations. These were then cluster analyzed, which was followed by model identification using representative genes in TNBC cell lines.

There is consensus that significant preprocessing, including background adjustment, normalization, and summarization, is required before a specific gene may be accurately assessed using a complied dataset [29]. Based on the published gene lists of the six subtypes of TNBC proposed by Lehmann et al. [7], using our compiled dataset, we found that there was clearly distinct subtype presentation among nonunion samples (Fig. 2, left panel), but this subtyping was not the same for the Taiwanese population (Fig. 2, right panel). Based on these finding, we renormalized the Taiwanese data using the MAS5 procedure and carried out clustering; this resulted in five rather than six clear subtypes being present in the Taiwanese population. Previous studies have suggested that the 

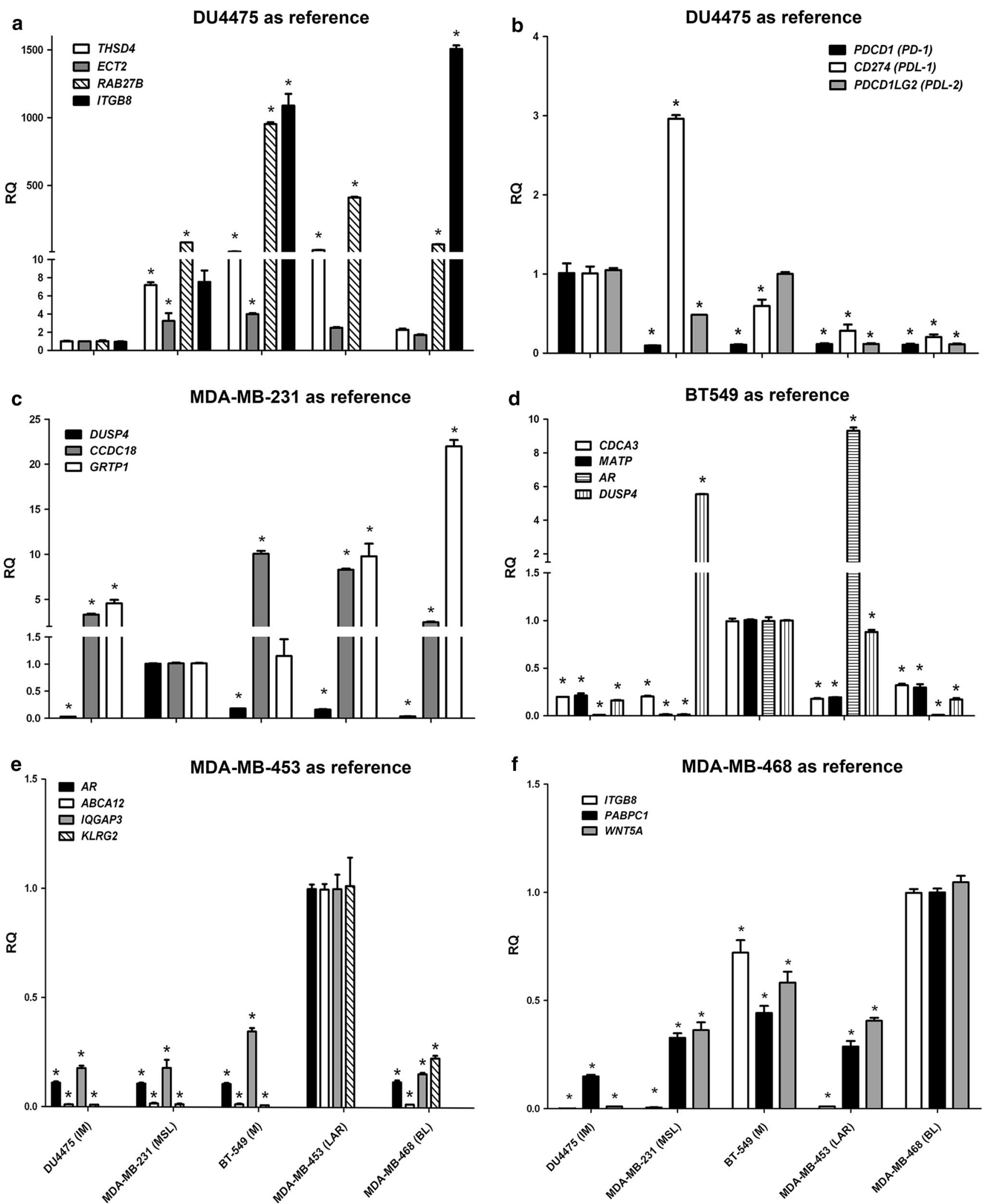
GCRMA approach might be responsible for introducing artifacts into the data analysis and that this can lead to a systematic overestimate of pairwise correlations within the data. In this context, it has been suggested that the MAS5 approach provides the most faithful cellular network reconstruction [30, 31].

Although from three to six TNBC subtypes have been proposed by various authors either using gene ontologies $[10,32]$, therapeutic targets $[11,12]$ or mRNA profiles as the diagnostic criteria [13], the exact number of TNBC subtypes that occur in women remains an open question [14]. Our findings identified five subtypes and these were the IM, MSL, M, LAR and BL subtypes. Interestingly, the BL1 and BL2 subtypes of the Lehmann's six type classification were clustered as a single BL subtype in our Taiwanese dataset. We attribute this discrepancy to a result of a smaller sample size, as the number of subtypes tends to increase with sample size.

Several lines of evidence suggest that the interactions of cancer cells with their microenvironment are a critical feature during tumor progression. The cell types involved in such interactions are not necessarily stromal cells [33], but also include macrophages [34], endothelial cells [35], and $\mathrm{T}$ cells [36]. Interestingly, we found significant upregulation of PDCD1 (PD1), CD274 (PDL1), and PDCD1LG2 (PDL2) expression in the IM subtype compared to the MSL subtype in our compiled dataset. However, when using DU4475 (IM) as the reference line, there was significant upregulation of PDCDI (PDI), and PDCD1LG2 (PDL2), but not of CD274 (PDL1), compared to MDA-MB-231 (MSL) (Supplementary Reference 3). We attribute this discrepancy to the study samples used, namely, cell lines versus tumor tissue. In the former, only cancer cells were investigated, while in the latter, cancer cells and other cells participating in the tumor microenvironment were investigated as a pool. It should be noted that the IM and MSL subtypes in our dataset share many canonical pathways, such as the iCOS-iCOSL signaling pathway (Table 4), which suggests the presence of significant similarity between these two subtypes. This seems to be supported by previous findings, which indicated that some transcripts present in the IM and MSL subtypes are contributed to by the tumor microenvironment [14].

The expression of the androgen receptor (AR) plays various different prognostic roles depending on the breast cancer subtype, such as the difference between ER-positive and ER-negative breast cancers with the expression levels of around 67-88\% [37, 38] and 12-50\% [39] for AR, respectively. Importantly in this context, it should be noted that the prevalence of $\mathrm{AR}$ expression has been found to range from $0-53 \%$ of TNBC [40].

In our compiled dataset, the percentages of the LAR subtype among nonunion and Taiwanese TNBC women were found to be 11.13 and $23.58 \%$, respectively. There is evidence suggesting that AR expression is about $60 \%$ among early breast cancers and is more frequently expressed in ER-positive than ER-negative breast cancers [41]. We speculate that ethnic differences might explain the variation in the percentage of the AR subtype between these different populations. However, further validation of this speculation is needed. If we examine cell line-specific gene expression, although the AR gene in BT-549 (M) is upregulated compared to DU4475 (IM), MDA-MB-468 (BL1) and MDA-MB-231 (MSL), the AR gene transcript in MDA-MB-453 (LAR) is ninefold higher than in BT-549 (M), which suggests that this change in AR gene expression is specific to the LAR subtype. Recent discrepancies concerning the role of AR have been noted in various TNBC basic and clinical studies and both AR agonist and AR antagonist clinical trials have been designed for the treatment of TNBC and $\mathrm{ER}^{+}$breast cancers [41-43]. Thus, the therapeutic role of $\mathrm{AR}$ remains an open question.

In summary, our findings suggest that there exist different presentations between nonunion and Taiwanese female populations in terms of TNBC subtypes. The fact that there seems to be correlation between the IM and MSL subtypes suggests the involvement of the tumor microenvironment in TNBC subtype classification might help to provide important information when selecting therapeutic targets or designing for clinical trials for TNBC patients.

Acknowledgements This work was supported by grants from Ministry of Health and Welfare (Center of Excellence for Cancer Research at Taipei Veterans General Hospital phase II, MOHW103TDU212114002, MOHW104TDUB211124001, MOHW105TDUB211134003, and Taipei Veterans General Hospital, (V106C029). We thank the Genome Research Center, National YangMing University, for the assistance on oligonucleotide microarray analysis and open array TaqMan ${ }^{\circledR}$ Gene Expression Assays.

\section{Compliance with ethical standards}

Conflict of interests I confirm that I have read BioMed Central's guidance on competing interests and have included a statement indicating that none of the authors have any competing interests in the manuscript.

Ethical approval Since this study was a retrospective review of medical records, it was approved by Institutional Review Board of Taipei Veterans General Hospital (\# 2013-10-020BC), and no patients' consent form was needed. The human triple negative breast cancer cell lines were used under the approval of Institutional Review Board of Taipei Veterans General Hospital (\# 2016-06-012BC).

Authors' contributions Tseng, LM and Chiu, JH contributed in forming the idea and writing the manuscript and providing clinical data. Chiu JH contributed in conducting the microarray analysis. Wang YL contributed in publicly available data acquisition. Liu CY contributed in providing cell lines. Tsai YF contributed in providing clinical data. Yang CW contributed in providing microarray analysis comments. Shyr YM contributed in providing clinical and facilities supports. 
Open Access This article is distributed under the terms of the Creative Commons Attribution 4.0 International License (http://crea tivecommons.org/licenses/by/4.0/), which permits unrestricted use, distribution, and reproduction in any medium, provided you give appropriate credit to the original author(s) and the source, provide a link to the Creative Commons license, and indicate if changes were made.

\section{References}

1. Tao Z, Shi A, Lu C, Song T, Zhang Z, Zhao J (2015) Breast cancer: epidemiology and etiology. Cell Biochem Biophys 72(2):333-338. doi:10.1007/s12013-014-0459-6

2. DeSantis C, Siegel R, Bandi P, Jemal A (2011) Breast cancer statistics, 2011. CA Cancer J Clin 61(6):409-418. doi:10.3322/ caac. 20134

3. Skibinski A, Kuperwasser C (2015) The origin of breast tumor heterogeneity. Oncogene 34(42):5309-5316. doi:10.1038/onc. 2014.475

4. Kumar P, Aggarwal R (2016) An overview of triple-negative breast cancer. Arch Gynecol Obstet 293(2):247-269. doi:10. 1007/s00404-015-3859-y

5. Podo F, Buydens LM, Degani H, Hilhorst R, Klipp E, Gribbestad IS, Van Huffel S, van Laarhoven HW, Luts J, Monleon D, Postma GJ, Schneiderhan-Marra N, Santoro F, Wouters H, Russnes HG, Sorlie T, Tagliabue E, Borresen-Dale AL, Consortium F (2010) Triple-negative breast cancer: present challenges and new perspectives. Mol Oncol 4(3):209-229. doi:10.1016/j.molonc.2010. 04.006

6. Kuo WH, Chang YY, Lai LC, Tsai MH, Hsiao CK, Chang KJ, Chuang EY (2012) Molecular characteristics and metastasis predictor genes of triple-negative breast cancer: a clinical study of triple-negative breast carcinomas. PLoS ONE 7(9):e45831. doi:10.1371/journal.pone.0045831

7. Lehmann BD, Bauer JA, Chen X, Sanders ME, Chakravarthy AB, Shyr Y, Pietenpol JA (2011) Identification of human triple-negative breast cancer subtypes and preclinical models for selection of targeted therapies. J Clin Investig 121(7):2750-2767. doi:10. 1172/JCI45014

8. Ahn SG, Kim SJ, Kim C, Jeong J (2016) Molecular classification of triple-negative breast cancer. J Breast Cancer 19(3):223-230. doi:10.4048/jbc.2016.19.3.223

9. Le Du F, Eckhardt BL, Lim B, Litton JK, Moulder S, MericBernstam F, Gonzalez-Angulo AM, Ueno NT (2015) Is the future of personalized therapy in triple-negative breast cancer based on molecular subtype? Oncotarget 6(15):12890-12908. doi:10. 18632/oncotarget.3849

10. Anders CK, Abramson V, Tan T, Dent R (2016) The evolution of triple-negative breast cancer: from biology to novel therapeutics. Am Soc Clin Oncol Educ Book Am Soc Clin Oncol Meet 35:34-42. doi:10.14694/EDBK_159135

11. Barton VN, D'Amato NC, Gordon MA, Lind HT, Spoelstra NS, Babbs BL, Heinz RE, Elias A, Jedlicka P, Jacobsen BM, Richer JK (2015) Multiple molecular subtypes of triple-negative breast cancer critically rely on androgen receptor and respond to enzalutamide in vivo. Mol Cancer Ther 14(3):769-778. doi:10.1158/ 1535-7163.MCT-14-0926

12. Burstein MD, Tsimelzon A, Poage GM, Covington KR, Contreras A, Fuqua SA, Savage MI, Osborne CK, Hilsenbeck SG, Chang JC, Mills GB, Lau CC, Brown PH (2015) Comprehensive genomic analysis identifies novel subtypes and targets of triplenegative breast cancer. Clin Cancer Res 21(7):1688-1698. doi:10.1158/1078-0432.ccr-14-0432
13. Liu YR, Jiang YZ, Xu XE, Yu KD, Jin X, Hu X, Zuo WJ, Hao S, Wu J, Liu GY, Di GH, Li DQ, He XH, Hu WG, Shao ZM (2016) Comprehensive transcriptome analysis identifies novel molecular subtypes and subtype-specific RNAs of triple-negative breast cancer. Breast Cancer Res 18(1):33. doi:10.1186/s13058-0160690-8

14. Lehmann BD, Jovanovic B, Chen X, Estrada MV, Johnson KN, Shyr Y, Moses HL, Sanders ME, Pietenpol JA (2016) Refinement of triple-negative breast cancer molecular subtypes: implications for neoadjuvant chemotherapy selection. PLoS ONE 11(6):e0157368. doi:10.1371/journal.pone.0157368

15. Lehmann BD, Pietenpol JA, Tan AR (2015) Triple-negative breast cancer: molecular subtypes and new targets for therapy. Am Soc Clin Oncol Educ Book Am Soc Clin Oncol Meet. doi:10. 14694/EdBook_AM.2015.35.e31

16. Brewster AM, Chavez-MacGregor M, Brown P (2014) Epidemiology, biology, and treatment of triple-negative breast cancer in women of African ancestry. Lancet Oncol 15(13):e625e634. doi:10.1016/S1470-2045(14)70364-X

17. Llanos AA, Chandwani S, Bandera EV, Hirshfield KM, Lin Y, Ambrosone CB, Demissie K (2015) Associations between sociodemographic and clinicopathological factors and breast cancer subtypes in a population-based study. Cancer Causes Control 26(12):1737-1750. doi:10.1007/s10552-015-0667-4

18. Martinez ME, Wertheim BC, Natarajan L, Schwab R, Bondy M, Daneri-Navarro A, Meza-Montenegro MM, Gutierrez-Millan LE, Brewster A, Komenaka IK, Thompson PA (2013) Reproductive factors, heterogeneity, and breast tumor subtypes in women of mexican descent. Cancer Epidemiol Biomarkers Prev 22(10):1853-1861. doi:10.1158/1055-9965.EPI-13-0560

19. Clarke CA, Keegan TH, Yang J, Press DJ, Kurian AW, Patel AH, Lacey JV Jr (2012) Age-specific incidence of breast cancer subtypes: understanding the black-white crossover. J Natl Cancer Inst 104(14):1094-1101. doi:10.1093/jnci/djs264

20. Lund MJ, Trivers KF, Porter PL, Coates RJ, Leyland-Jones B, Brawley OW, Flagg EW, O'Regan RM, Gabram SG, Eley JW (2009) Race and triple negative threats to breast cancer survival: a population-based study in Atlanta, GA. Breast Cancer Res Treat 113(2):357-370. doi:10.1007/s10549-008-9926-3

21. Dietze EC, Sistrunk C, Miranda-Carboni G, O'Regan R, Seewaldt VL (2015) Triple-negative breast cancer in AfricanAmerican women: disparities versus biology. Nat Rev Cancer 15(4):248-254. doi:10.1038/nrc3896

22. Chen LH, Kuo WH, Tsai MH, Chen PC, Hsiao CK, Chuang EY, Chang LY, Hsieh FJ, Lai LC, Chang KJ (2011) Identification of prognostic genes for recurrent risk prediction in triple negative breast cancer patients in Taiwan. PLoS ONE 6(11):e28222. doi:10.1371/journal.pone.0028222

23. Hicks C, Kumar R, Pannuti A, Backus K, Brown A, Monico J, Miele L (2013) An integrative genomics approach for associating GWAS information with triple-negative breast cancer. Cancer Inform 12:1-20. doi:10.4137/CIN.S10413

24. Wu Y, Sarkissyan M, Elshimali Y, Vadgama JV (2013) Triple negative breast tumors in African-American and Hispanic/Latina women are high in $\mathrm{CD} 44^{+}$, low in $\mathrm{CD} 24^{+}$, and have loss of PTEN. PLoS ONE 8(10):e78259. doi:10.1371/journal.pone. 0078259

25. Jacot W, Lopez-Crapez E, Thezenas S, Senal R, Fina F, Bibeau F, Romieu G, Lamy PJ (2011) Lack of EGFR-activating mutations in European patients with triple-negative breast cancer could emphasise geographic and ethnic variations in breast cancer mutation profiles. Breast Cancer Res 13(6):R133. doi:10.1186/ bcr3079

26. Chavez-Macgregor M, Liu S, De Melo-Gagliato D, Chen H, Do KA, Pusztai L, Fraser Symmans W, Nair L, Hortobagyi GN, Mills GB, Meric-Bernstam F, Gonzalez-Angulo AM (2014) 
Differences in gene and protein expression and the effects of race/ethnicity on breast cancer subtypes. Cancer Epidemiol Biomarkers Prev 23(2):316-323. doi:10.1158/1055-9965.EPI-130929

27. Tsai CH, Chiu JH, Yang CW, Wang JY, Tsai YF, Tseng LM, Chen WS, Shyr YM (2015) Molecular characteristics of recurrent triple-negative breast cancer. Mol Med Rep 12(5):7326-7334. doi: $10.3892 / \mathrm{mmr} .2015 .4360$

28. Chomczynski P, Sacchi N (1987) Single-step method of RNA isolation by acid guanidinium thiocyanate-phenol-chloroform extraction. Anal Biochem 162(1):156-159. doi:10.1006/abio. 1987.9999

29. Gautier L, Moller M, Friis-Hansen L, Knudsen S (2004) Alternative mapping of probes to genes for Affymetrix chips. BMC Bioinform 5:111. doi:10.1186/1471-2105-5-111

30. Irizarry RA, Bolstad BM, Collin F, Cope LM, Hobbs B, Speed TP (2003) Summaries of Affymetrix GeneChip probe level data. Nucleic Acids Res 31(4):e15

31. Lim WK, Wang K, Lefebvre C, Califano A (2007) Comparative analysis of microarray normalization procedures: effects on reverse engineering gene networks. Bioinformatics 23(13):i282i288. doi:10.1093/bioinformatics/btm201

32. Jezequel P, Loussouarn D, Guerin-Charbonnel C, Campion L, Vanier A, Gouraud W, Lasla H, Guette C, Valo I, Verriele V, Campone M (2015) Gene-expression molecular subtyping of triple-negative breast cancer tumours: importance of immune response. Breast Cancer Res 17:43. doi:10.1186/s13058-0150550-y

33. Nitsche U, Stangel D, Pan Z, Schlitter AM, Esposito I, Regel I, Raulefs S, Friess H, Kleeff J, Erkan M (2016) Periostin and tumor-stroma interactions in non-small cell lung cancer. Oncol Lett 12(5):3804-3810. doi:10.3892/ol.2016.5132

34. Hsu DS, Wang HJ, Tai SK, Chou CH, Hsieh CH, Chiu PH, Chen NJ, Yang MH (2014) Acetylation of snail modulates the cytokinome of cancer cells to enhance the recruitment of macrophages. Cancer Cell 26(4):534-548. doi:10.1016/j.ccell.2014. 09.002

35. Romero-Lopez M, Trinh AL, Sobrino A, Hatch MM, Keating MT, Fimbres C, Lewis DE, Gershon PD, Botvinick EL, Digman M, Lowengrub JS, Hughes CC (2017) Recapitulating the human tumor microenvironment: colon tumor-derived extracellular matrix promotes angiogenesis and tumor cell growth. Biomaterials 116:118-129. doi:10.1016/j.biomaterials.2016.11.034
36. Martinenaite E, Munir Ahmad S, Hansen M, Met O, Westergaard MW, Larsen SK, Klausen TW, Donia M, Svane IM, Andersen MH (2016) CCL22-specific T Cells: modulating the immunosuppressive tumor microenvironment. Oncoimmunology 5(11):e1238541. doi:10.1080/2162402X.2016.1238541

37. Loibl S, Muller BM, von Minckwitz G, Schwabe M, Roller M, Darb-Esfahani S, Ataseven B, du Bois A, Fissler-Eckhoff A, Gerber B, Kulmer U, Alles JU, Mehta K, Denkert C (2011) Androgen receptor expression in primary breast cancer and its predictive and prognostic value in patients treated with neoadjuvant chemotherapy. Breast Cancer Res Treat 130(2):477-487. doi:10.1007/s10549-011-1715-8

38. Hu R, Dawood S, Holmes MD, Collins LC, Schnitt SJ, Cole K, Marotti JD, Hankinson SE, Colditz GA, Tamimi RM (2011) Androgen receptor expression and breast cancer survival in postmenopausal women. Clin Cancer Res 17(7):1867-1874. doi:10.1158/1078-0432.CCR-10-2021

39. Gucalp A, Tolaney S, Isakoff SJ, Ingle JN, Liu MC, Carey LA, Blackwell K, Rugo H, Nabell L, Forero A, Stearns V, Doane AS, Danso M, Moynahan ME, Momen LF, Gonzalez JM, Akhtar A, Giri DD, Patil S, Feigin KN, Hudis CA, Traina TA, Translational Breast Cancer Research Consortium (2013) Phase II trial of bicalutamide in patients with androgen receptor-positive, estrogen receptor-negative metastatic breast cancer. Clin Cancer Res 19(19):5505-5512. doi:10.1158/1078-0432.CCR-12-3327

40. McNamara KM, Yoda T, Takagi K, Miki Y, Suzuki T, Sasano H (2013) Androgen receptor in triple negative breast cancer. J Steroid Biochem Mol Biol 133:66-76. doi:10.1016/j.jsbmb. 2012.08.007

41. Pietri E, Conteduca V, Andreis D, Massa I, Melegari E, Sarti S, Cecconetto L, Schirone A, Bravaccini S, Serra P, Fedeli A, Maltoni R, Amadori D, De Giorgi U, Rocca A (2016) Androgen receptor signaling pathways as a target for breast cancer treatment. Endocr Relat Cancer 23(10):R485-R498. doi:10.1530/erc16-0190

42. Asano Y, Kashiwagi S, Goto W, Tanaka S, Morisaki T, Takashima T, Noda S, Onoda N, Ohsawa M, Hirakawa K, Ohira M (2017) Expression and clinical significance of androgen receptor in triple-negative breast cancer. Cancers. doi:10.3390/ cancers 9010004

43. Narayanan R, Dalton JT (2016) Androgen receptor: a complex therapeutic target for breast cancer. Cancers. doi:10.3390/ cancers 8120108 